Infusionsther Transfusionsmed 1994;21:242-243

\title{
MEDICA 94
}

16.-19. November 1994- Düsseldorf- Messegelände

Kurzprogramm

ittwoch, 16, November 1994

Oonnerstag, 17. November 1994

9.15-12.30 Uhr

14.30-18.00 Uhr

9.15-12.30 Uhr

14.30-18.00 Uhr

Hätten Sie's besser gewußt?

Prof. Dr. O. Leiß, Wiesbaden

Bio-Äquivale $\cap z$ - überbewertet Oder entscheidendes Qualitätskriterium? Gemei̊nsame

Veranstaltung der Apothekerkammer Nordrhein und der Ärztekammer Nordrhein

Screening - Untersuchung!

Was ist sinnvoll - was ist gesichert - Vorsorge

fürdie Nachsorge!

Prof. Dr. E.-G. Loch,

Prof. Dr. O. Leiß, Wiesbaden 24

Bringt richtige Ernährung bessere Gesundhett'?

Prof. Dr. P. Schauder, Göttingen Prof. Dr. E.-G. Loch, Wiesbaden

Erfolgreiche Pharma Kommunikation-2. EPKO-Seminarkongress

Anmeldu $\cap$ g nur bei:

Berliner Medizinische Verlagsanstalt $\mathrm{GmbH}$

Clausewitzstr. 4, 10629 Berlin

Prävention, Diagnose und Therapie von Umwelterkra kungen Prof. Dr. Ingrid Gerhard,

Heidelberg

Darmflora in Symbiose und Pathogenität

Antibiotika - Probiotika

Prof. Dr. med. Dres, h. c. L. Demling,

Schlüsselfeld

Prof. Dr. rer. nat, B. Wiedemann, Bonn

Psychotherapie und Medikamente Prof. Dr. G. Nissen, Würzburg

MEDICA iuristica

Methoden der statistischen Wirtschaftlichkeits-

prüfung auf dem iuristischen Prüfstand

RA M. Broglie, Wiesbaden 5

Herausforderungen fürdie Internistische Praxis - Neue Wege in der Hepatologie 
Prof. Dr. W.-H. Schmiegel, Bochum

Gre zsituationen - Sterbebegleitung Prof. Dr. Waltraud Kruse, Aachen

MEDICA iuristica

Arzneimittelregresse und Abwehrstrategie

RA H. Wartensleben, Stolberg

EKG-GrundkursTeil I

Prof. Dr. K. D. Grosser, Krefeld

Dr. F.-R. Althoff, Viersen

Kampf dem Krebs - Immunprophyla×e, Diagnostik, Therapie

32

Prof. Dr. H. W. Baenkler, Erlangen

Zukunftssicheres Krankenhaus:

Klinik-Management u. -Marketing zur Opti-

mierung der intemen $u$. externen Kooperation

unter neuen Rahmenbedingungen

Prof. Dr. G. F. Riegl, Augsburg

27

EKG-GrundkursTeil II

Prof. Dr. K. D. Grosser, Krefeld

Dr. F.-R. Althoff, Viersen

Ethik in der Notfallmedizin Grenzprobleme im Notfall- u. Notarztdienst

Prof. Dr. P. Sefrin, Würzburg

MEDICA Oeconomica

Aktuelle Fragen aus der Gesundheitspoiitik

Prof. Dr. H. R. Vogel, Frankfurt 37

Arztpraxis 2000 - Wirtschaftliche Praxisplanung und -führung

Dr. K. Goder, Neuss

Demenz im Alter: Medizinische, ethische und ökonomische Problemlõsungen

Prof. Dr. B. Fischer, Nordrach-Klausenbach17

Problemkreis atopisches Ekzem - Lösungs-wege für Arzt und Patient

Prof. Dr. E. Schõpf, Freiburg

Zukunftssichere Arztpraxis: Praxis-Manage

ment $u$. -Marketing zur Patientenbetreuung

mit höherem Wirkungsgrad u. zur Verbesse-

rung der Berufsqualität für Praxis-Teams

Prof. Dr. G. F. Riegl, Augsburg 38

Polymerase-Kettenreaktion (PCR)

Chancen u. Gefahren molekularbiologischer

Diagnostik

Prof. Dr. J. D. Kruse-Jarres, Stuttgart 7

Das problematische Ulcus cruris Prof. Prof. h. c. Dr. N. Klüken, Essen

Teurere Arzneimittel - die billigere Alternative ? Kosten-Nutzen-Berechnung an praktischen

Beispielen Prof. Dr. P. Weidmann, Bern

HWS-Schulter-Arm-Syndrom Prot. Dr. A. Klûmper, Freiburg

Qualifikationsseminar: 24-Stunden-Blutdruckmessung 
Prof. Dr. B. Krönig, Trier

Diabetische Neuropathie PD Dr. D. Ziegler, Düsseldorf

30

Qualifikationsseminar:

24-Stunden-Blutdruckmessung

(Wiederholung)

Prof. Dr. B. Krönig, Trier

Lungenfunktionskurs für Arzthelferinnen und

MTAs

H. Wenske, Ingelheim40

Neues aus der Osteologie

Prof. Dr. Dr. E. Keck, Wiesbaden

Gibt es Neues bei der Therapie von urologischen Infektio en? $^{2}$

Prof. Dr. H.-J. Peters, Köln

Erkrankungen während der Schwa gerschaft -

Beratu $\mathrm{g}$ u. Behandlung im Praxisalltag des

Allgemeinarztes u. Internisten

Prof. Dr. O. Bartels, Nürnberg 31

Das Metabolische Syndrom - diagnostische u. therapeutische Konsequenzen für die Praxis -Prof.

Dr. R. Mies, Köln

Die einfache Lungenfunktionsdíagnostik für

Ãrzte - Theorie u. praktische Übungen für

Ärzte mit Vorkenntnissen

Dr. H. Fischer, Weilheim

10

Homöopathie in der Kinderheilkunde

Dr. M. Wiesenauer, Weinstadt

Management allergischer Erkrankungen vom

Typl

Dr. W. Kersten, Moers

Dr. J. Wenning, Villingen 26

Neurologisch-psychiatrischer Untersuchungskurs

Prof. Dr. B. Hofferberth, Coppenbrügge 42

Ärzte im Spannungsfeld zwischen Öffentlich-

keit, Politik und Industrie

Klaus Dallibor, Berlin 34 Do

Jodmangelkrankheiten in Deutschland: Diagnose, Therapie, Prophylaxe

Prof. Dr. F. A. Horster, Düsseldorf

Sind Allergie-Diag nostik und -Therapie noch

bezahlbar?

Prof. Dr. Dr. J. Ring, Hamburg

12

Hypertonie ' 94

Prof. Dr. V. Heimsoth, Damp

Pneumologie '94: Chronische Bronchitis und 
Lungenemphysem

Prof. Dr. D. Nolte, Bad Reichenhall 23

Die neue Abrechnung - die neue GoÄ -

Aktuelle Arzneimittel-Richtlinie $\cap$ - Negativlisten

- Positivlisten, Fachgruppenvergleiche

Dr. H. Massing, Ibbenbüren

Dr. J. Linden, Altena 33

Theorie und Praxis der Tumorschmerz-behandlung

Dr. R. Sittl, Erlangen in Zusammenarbeit mit

der Internat. Home-Care Akademie 43

Infektionen des Respirationstraktes Dr. H. Scholz, Berlin

Halle 6 Eingang Nord

Halle 6 Eingang Moid 